\title{
Theory does not meet experiment: transient dynamics changes patterns of exclusion in an intraguild predation system
}

\author{
Gabriel Andreguetto Maciel ${ }^{1}$ (D) $\cdot$ Roberto André Kraenkel $^{1}$
}

Received: 6 June 2017 / Accepted: 12 October 2017 / Published online: 13 November 2017

(C) The Society of Population Ecology and Springer Japan KK 2017

\begin{abstract}
Although empirical verifications of ecological theory are essential for the advance of our understanding of ecosystems functioning, they are often hard to obtain or even impractical. In this work we perform a detailed analysis of unexpected results found in a previous test of intraguild predation (IGP) theory. When the IG prey is the stronger competitor the IGP theory predicts a clear dynamical pattern along a resource gradient. In particular, the IG predator is expected to be excluded at low resources. In the experiment we analyze, IG prey was excluded at a resource level where the IG predator should be eliminated. We use a simple IGP model parametrized using mainly preliminary tests of the experiment. We suggest that experiment and theory agree if we look to the transient dynamics instead of asymptotic states, in which the usual theory is based. We show that extremely low IG prey populations during the transient may drive it to extinction and prevent the system from reaching long-term states. Our results are shown to be robust with respect to changes in initial conditions and parameters.
\end{abstract}

Keywords Community modules $\cdot$ Population dynamics . Test of ecological theory $\cdot$ Transient exclusion

\section{Introduction}

Intraguild predation (IGP) is a widespread phenomenon and occurs when two consumer species sharing a common resource, and hence competing, also engage into predation

Gabriel Andreguetto Maciel

maciel@ift.unesp.br

1 Instituto de Física Teórica, São Paulo State University (UNESP), São Paulo, Brazil
(Polis et al. 1989; Polis and Holt 1992). The consumer which prey on its competitor is referred to as the intraguild predator (IG predator), while the other is called intraguild prey (IG prey). Because the IG predator can prey on more than one trophic level, IGP is a subset of omnivory (Pimm and Lawton 1978).

Only recently IGP has received greater attention in the ecological literature. This lack of interest in the past has been attributed to the belief that IGP would be rare in nature, once studies pointed the destabilizing effect of omnivory on communities (Mylius et al. 2001). However, it has become now clear that IGP is ubiquitous in nature (Arim and Marquet 2004). These findings have motivated a number of theoretical studies that look for conditions under which consumers can coexist in these systems.

Polis and Holt (1997) set a formal theoretical framework for IGP and showed that for coexistence to be possible the IG prey must be the best at exploiting the resource. IGP combines elements of competition and predation and coexistence arises via trade-offs in the consumers habilities for consuming their competitor and the shared resource (Amarasekare 2010). Furthermore, these authors exposed the rich variety of resulting dynamics found in this system, including the potential existence of alternative states. Assuming the IG prey is the stronger competitor, when the environmental productivity is low theory predicts that the IG prey suppresses the IG predator, which expresses the fact that when resource level is low the competitive ability is decisive. Coexistence is restricted to intermediate productivities and when the environment is rich the IG predator eliminates the IG prey (Polis and Holt 1997; Mylius et al. 2001).

These results are quite general and pose a clear picture of the patterns of exclusion along a productivity gradient. However, so far only few attempts have been made to empirically verify these patterns. Diehl and Feissel (2001) 
in an experiment with bacteria (shared resource) and the ciliates Tetrahymena pyriformis (IG prey) and Blepharisma americanum (IG predator) verified the counterintuitive theoretical result that as resource productivity increases the IG prey population declines while the IG predator population increases. This same result was verified in Borer et al. (2003) in a field system consisting of a scale insect and its two specialist parasitoids, Aphytis melinus (the IG predator) and Encarsia perniciosi (the IG prey). Morin (1999) observed the exclusion of the ciliate B. americanum (IG predator in this case) by the ciliate Colpidium striatum (IG prey) when resource abundance was low. In higher productivities these consumers coexisted for many generations, indicating a shift from IG prey dominance to coexistence as theoretically predicted.

Montserrat et al. (2008) tested the IGP theory in a system consisting of two predatory mites feeding on cattail pollen. Although this experiment was designed to test the theory and its premises were confirmed to hold, such as the competitive superiority of the IG prey, results found are inconsistent with theoretical predictions. In productivities in which it was expected either coexistence or the elimination of the IG predator, for most replicates the IG prey was eliminated. In this work we focus on the analysis of this experiment. We introduce a simple model for IGP with chemostat resource growth as a representation of the system. We show that if we reinterpret results from theory, by analyzing the dynamics during transients, experiment and theory are consistent.

Studies on population dynamics have traditionally focused on equilibrium dynamics (Hastings 2004). This tendency can be easily understood due to the complexity of most systems of importance. These systems often present coupled nonlinear differential equations and analytical solutions can be found only in limited cases. Analysis of stationary states, in turn, has been extensively employed as a powerful tool for assessing analytical results and, in numerous cases, successfully captures important features of real systems.

Yet in many cases, in natura or in laboratory, systems may have not reached equilibrium and analysis of the transient dynamics is important (Hastings 2001). This is what occurs in many experiments in IGP, in which duration of observations are short relative to the generation time of species involved (Briggs and Borer 2005). Care must also be taken when systems present large oscillations during transients, once populations can attain very low levels so that the only reasonable interpretation is that extinction is likely, although stationary states do not predict exclusion of individuals (Hastings 2001).

As will be shown in this work, models for intraguild predation potentially present large oscillations in shortterm dynamics. We recall here that classical results of IGP are based on equilibrium states. If we take the transient dynamics fully into account, we can then formulate a new interpretation of the theory that strongly affects the expected exclusion patterns.

In particular, in the experiment of Montserrat et al. (2008) with mites, in productivity levels where it was expected either IG predator elimination or coexistence, the IG prey was first eliminated followed by the decline of the IG predator. Based on our theoretical framework we show that: although resulting stationary states in fact indicate coexistence, in the short-term dynamics the IG prey population attains very low levels, that are usually less than the unity. If we reinterpret this low population sizes as real extinctions then agreement between theory and experimental results is found.

To make this presentation self-contained we give a more detailed description of the experiment with mites in the next section. We then introduce the model for this system and the parameters used in simulations. Parameters were either estimated from preliminary results of the experiment or taken from other sources. Details of parameters estimation are given in the "Appendix 1". In the "Results", we show how IGP theory, given the parameters for these species, can be applied in this system. Finally, we then present the "Discussion".

\section{The experiment with mites}

Montserrat et al. (2008) tested IGP theory in a system composed of two predatory mite species, Iphiseius degenerans (IG predator) and Neoseiulus cucumeris (IG prey), feeding on cattail pollen (the shared resource). The generation time of these mite species is approximately 9 days. As IGP theory assumes the IG predator attacks the IG prey with a fixed attack rate even in the presence of resource and, in addition, non trivial patterns of exclusion along a productivity gradient arise only if the IG prey is the best competitor, preliminary tests were carried out before studying the IGP dynamics.

In order to verify the occurrence of intraguild predation in the presence of resource, one adult female of I. degenerans was exposed to 30 juvenile of $N$. cucumeris in presence and absence of resources. The number of dead juveniles and eggs laid by the female were counted after $24 \mathrm{~h}$. Arenas with the IG prey and resource and IG prey alone were used as controls for natural mortality. Results indicated that predation of the IG predator on the IG prey is not significantly affected by the presence of resource.

The competitive superiority of the IG prey was checked after evaluation of the dynamics of each consumer alone with resource. Two levels of pollen input rate were set ("high" and "intermediate") and only the IG prey persisted in the two levels. The IG predator persisted for high levels of 
pollen supply, but went excluded in the intermediate level. Because the IG prey can persist in lower pollen levels than the IG predator, the IG prey is the best at exploiting the resource (Tilman 1990).

The same "high" and "intermediate" levels of pollen used in preliminary tests were again used in the experiments on the dynamics of intraguild predation of this system. Ten gravid females of each consumer were added to the arena and initially were kept separated by a strip of filter paper. Six days later this strip was removed and competitors could then interact. Twice a week individuals were counted and pollen was added to the arena.

In the high level pollen supply, the IG predator excluded the IG prey in most of replicates and then persisted with resource. This result indicate that, in fact, this "high pollen" level supply falls in the region of IG predator dominance predicted by theory. Unexpectedly, however, extinction of the IG prey followed by the IG predator elimination was observed in the intermediate level. Because the IG predator can not persist with resource only in this pollen level, as verified in preliminary tests, either coexistence of consumers or the elimination of the IG predator was expected. Montserrat et al. (2008) suggested that transient dynamics, stochastic events and stage structure of populations may be responsible for these inconsistent results. Nevertheless, these authors did not carry detailed analysis on these propositions. In what follows, we will present an explanation based on the transient dynamics of this system.

\section{The model}

Because pollen (the basal resource in the experiments) was supplied at a constant inflow rate, a chemostat resource growth equation in the absence of consumers best resembles experimental conditions. Hence, we model the dynamics of the IGP system by the set of differential equations:

$$
\frac{\mathrm{d} P}{\mathrm{~d} t}=b^{\prime} a^{\prime} R P+\beta \alpha N P-m^{\prime} P
$$

$\frac{\mathrm{d} N}{\mathrm{~d} t}=b a R N-\alpha P N-m N$

$\frac{\mathrm{d} R}{\mathrm{~d} t}=\mu-\phi R-a N R-a^{\prime} P R$,

where $P$ and $N$ are respectively the populations of $I$. degenerans (IG predator) and of $N$. cucumeris (IG prey). $R$ is the amount of pollen. Consumers are described by the total number of individuals in the arenas and the amount of pollen is given by its total mass in mg. $\mu$ is the inflow rate of resources and $\phi$ is its decay rate. Once resource intake was controlled experimentally, $\mu$ can be obtained directly from experimental conditions. Consumers natural death rates are given by $m^{\prime}$ and $m . a^{\prime}$ and $a$ are the attack rates of the IG predator and the IG prey on the resource, respectively. Conversion rate of resources into IG predator (IG prey) population is given by $b^{\prime}(b)$. Finally, $\alpha$ gives the attack rate of $I$. degenerans on $N$. cucumeris and $\beta$ is the conversion rate of this consumption.

We do not intend to mimic the exact dynamics observed in experiments. Our purpose, however, is to call attention for the fact that transient dynamics in IGP often presents prey populations that attain levels very close to extinction and that applying these arguments we get a plausible explanation for the unexpected results found in Montserrat et al. (2008). Parameters used in our simulations are shown in Table 1. They were estimated using results from the experiment and taken from van Rijn et al. (2002) (see "Appendix 1").
Table 1 Parameters used in simulations

\begin{tabular}{llll}
\hline Parameter & Value & Unit & Description \\
\hline$b^{\prime}$ & 25.95 & Predator $/ \mathrm{mg}$ & Resource conversion efficiency \\
$b$ & 25.95 & Prey/mg & - \\
$a^{\prime}$ & 0.00037 & 1 (predator $\times$ day) & Resource attack rate \\
$a$ & 0.012 & 1 (prey $\times$ day) & - \\
$m^{\prime}$ & 0.05 & 1 day & Natural mortality rate \\
$m$ & 0.05 & 1 day & - \\
$\beta$ & 0.075 & Predator/prey & Consumer conversion rate \\
$\alpha$ & 0.1 & 1 (predator $\times$ day) & Consumer attack rate \\
$\mu$ & $1.37($ high $)$ & mg/day & Resource input rates \\
$\phi$ & $0.229($ intermediate) & mg/day & \\
\hline
\end{tabular}




\section{Results}

We integrate numerically the system of ordinary differential equations (Eqs. 1a-c) using Python odeint library. This library uses lsoda solver from the FORTRAN library odepack. For high pollen supply, both IG predator and IG prey grow and attain a stable state in simulations when they grow alone with resource, as shown in Figs. 1a and 2a. For intermediate pollen supply the IG prey again grows and attain a stationary state (Fig. 2b) while in this case the IG predator declines to zero (Fig.1b). Simulations therefore agree with preliminary experiments. These results simply reflect the parametrization procedure we used. Parameters related to resource consumption were estimated matching stationary states of equations to the population sizes observed for large times in experiments with consumers growing alone with resource. Initial populations and pollen level were set to match experimental conditions.

Figure 3 shows the simulated population dynamics at high level of pollen supply when the two consumer species are initially present. As verified experimentally, IG predator eliminates the IG prey at this resource level. The exact initial
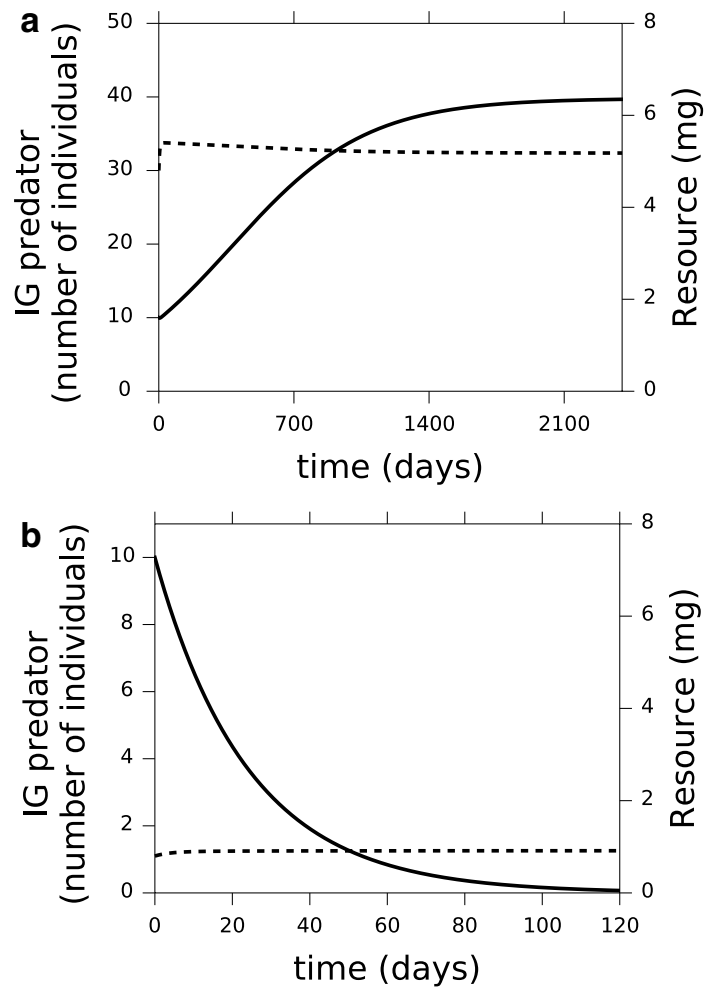

Fig. 1 Dynamics of the model (Eqs. 1a-c) when only $P$ (solid lines) and $R$ (dashed lines) are present for: a high pollen supply and $\mathbf{b}$ for intermediate pollen supply. IG predator does not persist in the intermediate resource level. Initial predator population was set to $P_{0}=10$ individuals in both panels. Initial resource levels are $R_{0}=4.8 \mathrm{mg}$ in a and $R_{0}=0.8 \mathrm{mg}$ in $\mathbf{b}$
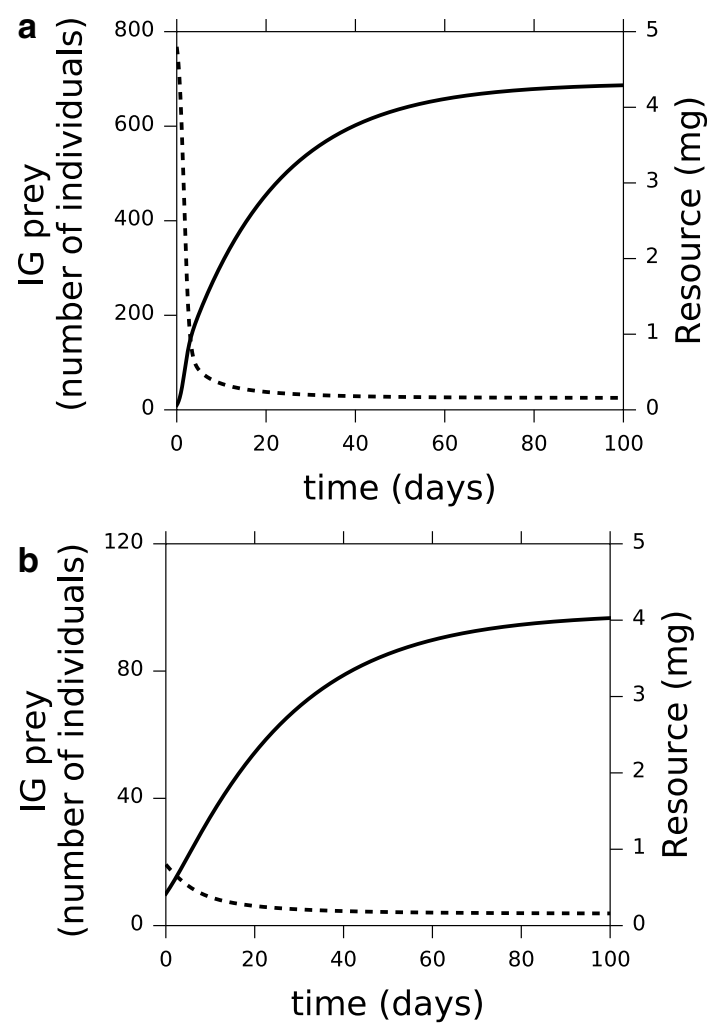

Fig. 2 Dynamics of the IG prey (solid lines) when it grows alone with resource (dashed lines). IG prey can persist at high (a) and intermediate (b) levels of resource, showing its superiority at exploiting the resource. We have $R_{0}=4.8 \mathrm{mg}$ in $\mathbf{a}$ and $R_{0}=0.8 \mathrm{mg}$ in $\mathbf{b}$. In both panels $N_{0}=10$

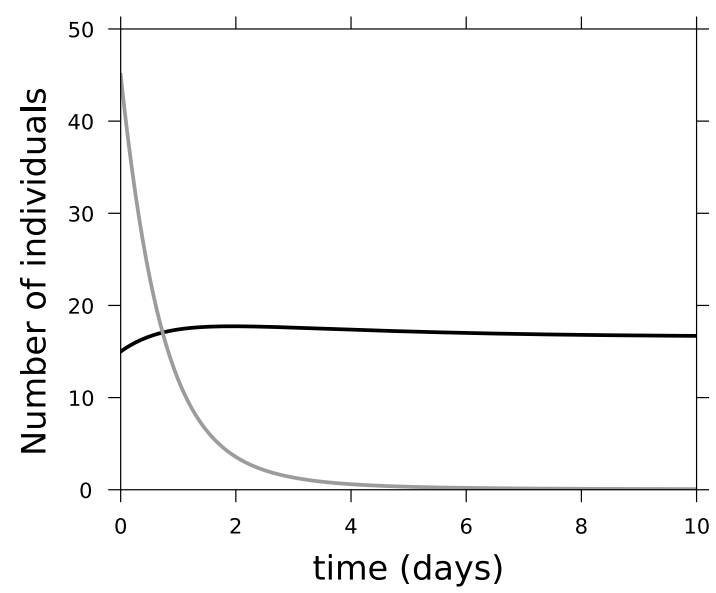

Fig. 3 Population dynamics for high pollen supply. IG predator and IG prey populations are represented by the black and gray lines, respectively. Parameters are shown in Table 1. Initial conditions are: $P_{0}=15, N_{0}=45$ and $R_{0}=4.8$ 


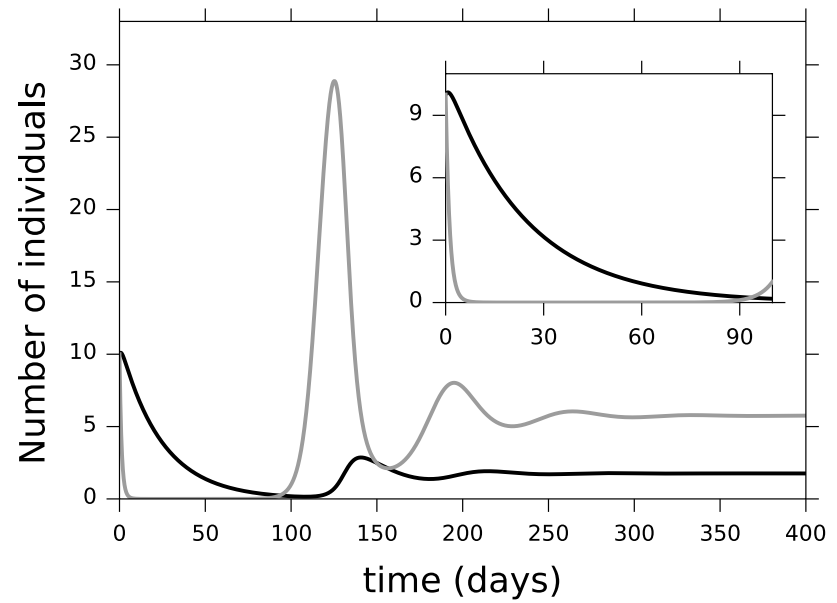

Fig. 4 Dynamics of $N$. cucumeris (gray line) and I. degenerans (black line) at intermediate pollen supply. The inset gives an enlarged view of the transient dynamics. Initial conditions are: $P_{0}=10$, $N_{0}=10$ and $R_{0}=0.8$

conditions in the experiments are variable in this case, as each consumer grew alone with resource for 6 days before they were allowed to interact. This result (elimination of IG prey), however, is not sensible to initial conditions.

We now look at intermediate pollen supply, where unexpected outcomes were verified in experiments. A typical dynamics found in simulations for this resource level is shown in Fig. 4. Let us recall that it was expected either coexistence or elimination of the IG predator. Our simulations show that populations converge to a stable coexistence state in an oscillatory fashion. Accordingly, the eigenvalues of the Jacobian matrix at the coexistence state are complexconjugate with negative real part (Hirsch et al. 2013). This state is therefore a stable focus.

In the short-term, the dynamics is characterized by a sudden decline of IG prey population, as shown in the inset of Fig. 4. Its population attains extremely low levels, which represents an actual elimination in a real population. The IG predator population also initially declines, but as the IG prey is re-established it grows to its stationary state. We ran simulations with other initial conditions that are similar to conditions in some replicates in the experiments and the qualitative results of Fig. 4 do not change (see "Appendix 2").

However, there are initial conditions at intermediate pollen supply for which the IG prey is not driven to extremely small values. In fact, given that the long-term dynamics in our simulations shows a stable coexistence, initial conditions close to the equilibrium states would not reproduce the dynamics here explored. We perform now a full investigation on how initial conditions of the IG predator $\left(P_{0}\right)$ and the IG prey $\left(N_{0}\right)$ affect the transient dynamics. We numerically solve the dynamical system for different combinations of $P_{0}$ and $N_{0}$ and check for transient elimination. As we are modeling absolute number of individuals, we interpret IG prey populations lower than the unity as an actual elimination. The initial conditions that do not lead to transient elimination are shown in the gray region of Fig. 5. The two panels correspond to different initial conditions of basal resource. Note that elimination of the IG prey does not occur only for a restricted range of initial IG predator populations and this region decreases as $R_{0}$ is increased. These values of $P_{0}$ are below the conditions set in the experiments. Our arguments are then robust to variations in initial conditions. In principle, experiments with starting populations in the gray region of Fig. 5 could be performed to test if the IG prey is eliminated or not. However, there could be some limitations in this case due to the low initial number of IG predator individuals.
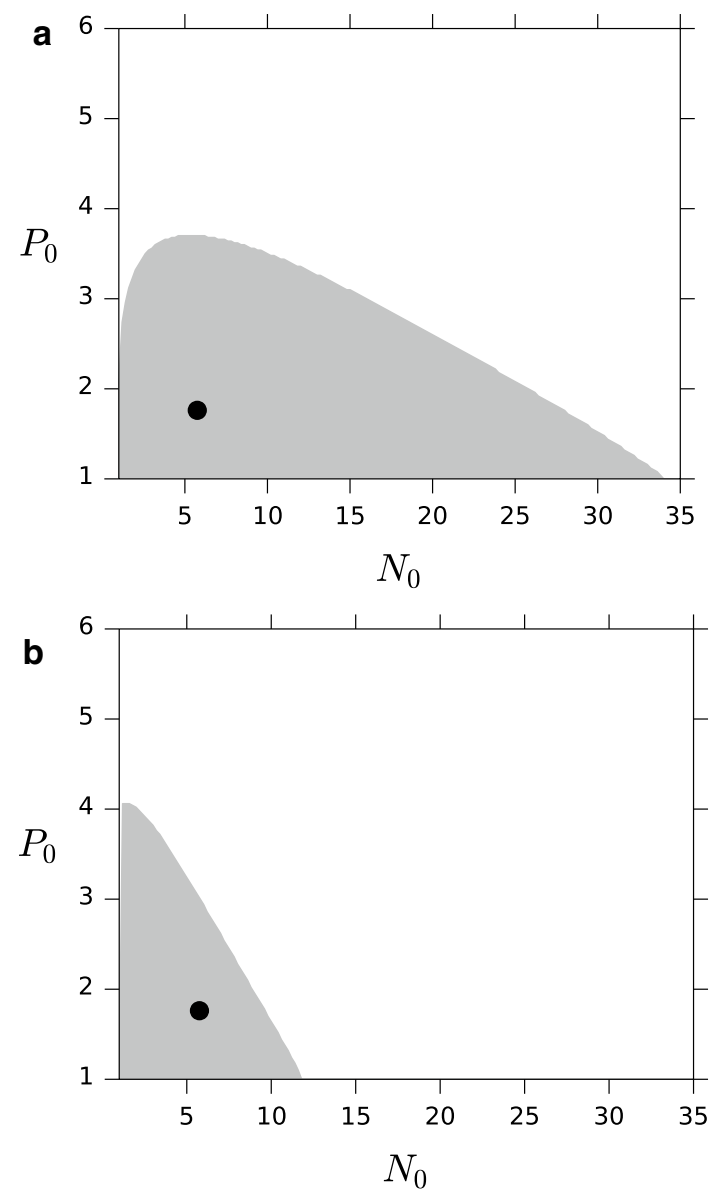

Fig. 5 Resulting transient dynamics as a function of IG predator $\left(P_{0}\right)$ and IG prey $\left(N_{0}\right)$ initial conditions. Gray region indicates initial conditions for which the IG prey population is not driven to values less than the unity. Therefore, according to our interpretation no actual elimination occurs for these initial conditions. The black dot indicates the position of the coexistence steady state. We set $R_{0}=0.8$ in $\mathbf{a}$ and $R_{0}=2.4 \mathrm{in} \mathrm{b}$. Parameters are those of Table 1 and resource input rate is set to intermediate 


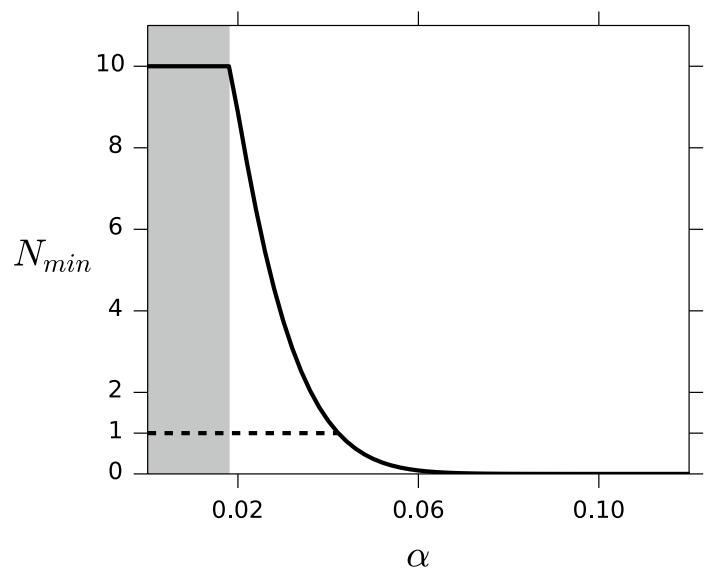

Fig. 6 Minimum population attained by the IG prey during transient as a function of IG predator attack rate. The gray region indicates $\alpha$ values for which IG prey eliminates the IG predator. Pollen supply is set to the intermediate level. Other parameters are fixed to values of Table 1. Initial conditions are: $P_{0}=N_{0}=10$ and $R_{0}=0.8$

The rate in which the IG predator attacks the IG prey also greatly influences the transient dynamics. In Fig. 6, we track how the minimum population size attained by the IG prey during transients $\left(N_{\text {min }}\right)$ depends on $\alpha$. This minimum is detected from the numerical solutions of the system of equations. For low $\alpha$ the IG prey eliminates the IG predator and, once the equilibrium size of IG prey in presence of pollen only is greater than 10 (our initial conditions used), $N_{\text {min }}$ is simply the initial value, as shown in the gray region of Fig. 6. Then, as $\alpha$ increases and the long-term dynamics becomes coexistence, $N_{\min }$ is a fast decreasing function of $\alpha$. This figure also shows that the minimum attack rate for an actual transient elimination is rapidly attained.

\section{Discussion}

Tests of theory are essential for the advance of our understanding of ecological processes. The experiment of Montserrat et al. (2008) provides a great opportunity to test IGP theory. We have explicitly shown in this work how IGP theory applies to that experiment with mites.

The dynamics for intermediate pollen supply with parameters of this system is predicted to have a coexistence stable state asymptotically, as shown in Fig. 4. This state is a stable focus and, accordingly, decaying oscillations of populations are observed near the fixed point. In the experiments, the IG prey is excluded followed by the decline in the IG predator population for this level of resource. Given the continuous nature of differential equations and as the IGP theory is based on equilibrium states, the fact that the IG predator can not sustain its population with resource only at this level of pollen makes coexistence or IG predator elimination the only possible outcomes predicted by the usual theory.

Nevertheless, in the initial phase of the dynamics the IG prey population is driven to very low levels, as shown in the inset of Fig. 4. Hence, instead of looking to stationary states, in which usual theory is based, we reinterpret results assuming that extinction occurred during the transient. In this way the discrepancy between theory and experiment is overcome.

Considerations about population sizes during transients and the consequent risk of local extinctions had been reported before in a number of studies (Rosenzweig and MacArthur 1963; Rosenzweig 1971; Shulenburger et al. 1999; Noonburg and Abrams 2005; Steiner et al. 2012). But, as far as we know, this is the first study which examines the possibility of transient extinction in an intraguild predation system.

In the experiments there are signs of demographic stochasticity in the dynamics. For example, for high pollen supply there is one replicate where the IG predator is eliminated and at intermediate resource level the IG prey excludes the predator in one of the replicates [Figs. 3 and 4 of Montserrat et al. (2008)]. Times to extinction also vary between replicates. However, we do not have enough information for a full analysis of the stochastic population processes as the number of realizations of each experiment is very low. An important point in our study is that even with these considerations the deterministic model of continuous populations does not need to be abandoned. It captures the initial depression of IG prey population caused by the IG predator which we argue is the main driver of transient elimination.

We have provided therefore a robust and comprehensive explanation of the outcomes in this intraguild predation system. Consideration of transient dynamics is found to be crucial for understanding the experimental dynamics. In addition, there is no other apparent feature that could explain the fact that the IG predator eliminates the IG prey in productivities in which it can not sustain its population with resource only. Confrontations of empirical data with population dynamics models should then be careful when using only results from asymptotic analysis.

Acknowledgements The authors thank the Brazilian funding agencies CAPES, CNPq and FAPESP for financial support. We also thank André M. de Roos and Renato M. Coutinho for useful comments and suggestions on this work.

\section{Appendix 1}

In this appendix we give a detailed description about the estimation of parameters used in simulations. 


\section{Resource growth}

Inflow rates $(\mu)$ can be directly obtained from experimental procedures. For all resource levels twice a week new amounts of pollen were introduced in the arenas, $4.8 \mathrm{mg}$ for high pollen level experiments and $0.8 \mathrm{mg}$ for the intermediate level. $\mu$ can then be calculated by

$\mu=($ mg pollen $) \times \frac{2}{7 \text { day }}$.

Then we have:

$\mu=\left\{\begin{array}{l}1.37 \mathrm{mg} / \mathrm{day} \text { (high) } \\ 0.229 \mathrm{mg} / \mathrm{day} \text { (intermediate) . }\end{array}\right.$

Parameter $\phi$ is the rate in which pollen leaves the system if it is not consumed by the competitors. In principle, it would refer to the rate that pollen rots away. However, every time new pollen was added to the arenas they also removed remained grains. This factor ends up being determinant for estimation of this parameter.

According to Eq. 1c, without pollen reposition or consumption pollen decays exponentially at a rate $\phi$. Thus, $1 / \phi$ is the time needed for $64 \%$ of initial pollen to be depleted. Here we consider this time being 4 days, what gives

$\phi=0.25$ day $^{-1}$.

\section{Resource consumption abilities}

Initially, we fix natural mortality rates of consumers using parameter for I. degenerans taken from van Rijn et al. (2002) and attributing this same value for $N$. cucumeris. We have

$m^{\prime}=m=0.05 /$ day .

Experiments performed with consumers growing alone with resource were used to estimate parameters of resource consumption $\left(b^{\prime}, b, a^{\prime}\right.$ and $\left.a\right)$. For the IG prey, in both pollen levels (intermediate and high) its population grew and we assume it reached a stationary state that we will call $N_{h}^{*}$ and $N_{i}^{*}$ for high and intermediate levels, respectively. In presence of only $N$ and $R$, stationary states of Eqs. 1a-c can be easily obtained. Denoting $\mu_{h}$ and $\mu_{i}$ the high and intermediate inflow rates, respectively, stationary states of the IG prey for these two levels are

$$
\begin{aligned}
& N_{h}^{*}=\frac{\mu_{h} b}{m}-\frac{\phi}{a} \\
& N_{i}^{*}=\frac{\mu_{i} b}{m}-\frac{\phi}{a} .
\end{aligned}
$$

Using observed values of $N_{h}^{*}$ and $N_{i}^{*}$ and the other constants already determined, we can solve this system of equations and determine $b$ and $a$. This gives

$b=25.95$ prey $/ \mathrm{mg}$ and $a=0.012$ (prey $\times$ day) ${ }^{-1}$.

The IG predator, in turn, persisted only in the high pollen level supply, and then we have only one "stationary state" of reference $\left(P_{h}^{*}\right)$. We take $b^{\prime}$ as being equal to $b$ and $a^{\prime}$ is calculated matching the equilibrium state of the equations with the observed "equilibrium". The IG predator equilibrium point in the presence of pollen only is given by

$P_{h}^{*}=\frac{\mu_{h} b}{m^{\prime}}-\frac{\phi}{a^{\prime}}$.

Thus we have

$a^{\prime}=\frac{\phi}{\frac{\mu_{h} b}{m^{\prime}}-P_{h}^{*}}=0.00037(\text { predator } \times \text { day })^{-1}$.

\section{$\beta$ and $\alpha$}

During preliminary tests, Montserrat et al. (2008) exposed one female of the IG predator to 30 young individuals of IG prey. The number of dead juveniles in one day $\left(d_{P}\right)$ as well as the number of eggs laid by the IG predator (e) were counted. They also measured the number of dead juveniles in the absence of the IG predator $(d)$, so that we can calculate $\beta$ as

$\beta=\frac{e}{d_{P}-d}=0.075$ predator $/$ prey.

Finally, $\alpha$ can be estimated by calculating the fraction of the IG prey that was killed by the IG predator in one day. This results in $\alpha=0.33$. However, as in these tests the IG predator was exposed to juveniles only, which is the most vulnerable class of the IG prey and in the experiments all classes are present, this number gives only an upper limit. We use in this work

$\alpha=0.1$ (predator $\times$ day) $^{-1}$.

In the results section we present a sensibility analysis and show that our results are robust with respect to changes in this parameter.

\section{Appendix 2}

In Fig. 7 we show the dynamics of the intraguild predation for intermediate pollen supply, as in Fig. 4, using different initial conditions. The initial conditions in the experiment are variable because ten individuals of each species grow with resource only for 6 days until they are allowed to interact. In panel (a) we reproduce the case where IG prey 

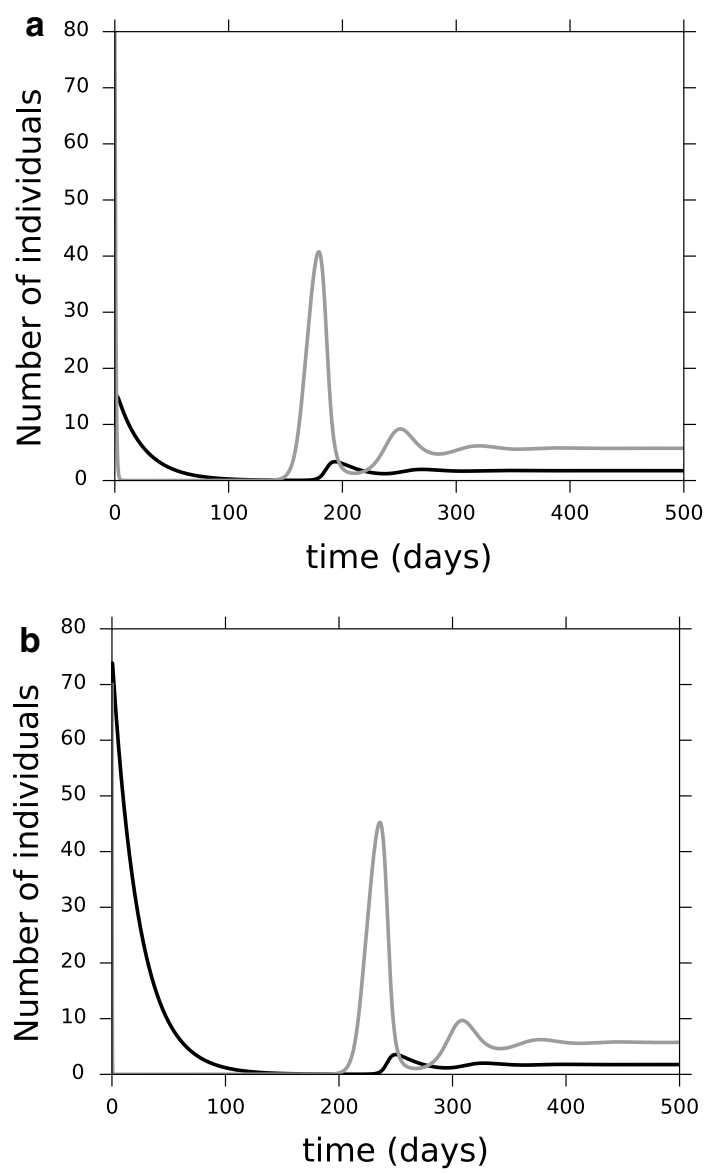

Fig. 7 Dynamics of the IG predator (black lines) and IG prey (gray lines) for intermediate pollen supply. Initial conditions are: $P_{0}=10$ and $N_{0}=80$ in a and $P_{0}=N_{0}=70$ in $\mathbf{b}$. In both panels $R_{0}=0.8$

population is initially larger than the IG predator. In panel (b) both populations are initially equal but larger than 10 . In both cases the IG prey population is rapidly driven to very low levels.

\section{References}

Amarasekare P (2010) Chap 2. Spatial dynamics of multitrophic communities. In: Cantrell S, Cosner C, Ruan S (eds) Spatial ecology. Chapman and Hall, Boca Raton, pp 15-31

Arim M, Marquet PA (2004) Intraguild predation: a widespread interaction related to species biology. Ecol Lett 7:557-564
Borer ET, Briggs CJ, Murdoch WW, Swarbrick SL (2003) Testing intraguild predation theory in a field system: does numerical dominance shift along a gradient of productivity. Ecol Lett 6:929-935

Briggs CJ, Borer ET (2005) Why short-term experiments may not allow long-term predictions about intraguild predation. Ecol Appl 15(4):1111-1117

Diehl S, Feissel M (2001) Intraguild prey suffer from enrichment of their resources: a microcosm experiment with ciliates. Ecology 82(11):2977-2983

Hastings A (2001) Transient dynamics and persistence of ecological systems. Ecol Lett 4:215-220

Hastings A (2004) Transients: the key to long-term ecological understanding? Trends Ecol Evol 19(1):39-45

Hirsch MW, Smale S, Devaney RL (2013) Differential equations, dynamical systems, and an introduction to chaos, 3rd edn. Elsevier Academic Press, Waltham

Montserrat M, Magalhes S, Sabelis MW, de Roos AM, Janssen A (2008) Patterns of exclusion in an intraguild predator-prey system depend on initial conditions. J Anim Ecol 77:624-630

Morin P (1999) Productivity, intraguild predation, and population dynamics in experimental food webs. Ecology 80(3):752-760

Mylius SD, Klumpers K, de Roos AM, Persson L (2001) Impact of intraguild predation and stage structure on simple communities along a productivity gradient. Am Nat 158(3):259-276

Noonburg EG, Abrams PA (2005) Transient dynamics limit the effectiveness of keystone predation in bringing about coexistence. Am Nat 165(3):322-335

Pimm SL, Lawton JH (1978) On feeding on more than one trophic level. Nature 275:542-544

Polis GA, Holt RD (1992) Intraguild predation: the dynamics of complex trophic interactions. Trends Ecol Evol 7:151-155

Polis GA, Holt RD (1997) A theoretical framework for intraguild predation. Am Nat 149(4):745-764

Polis GA, Holt RD, Myers CA (1989) The ecology and evolution of intraguild predation: potential competitors that eat each other. Annu Rev Ecol Syst 20:297-330

Rosenzweig ML (1971) Paradox of enrichment: destabilization of exploitation ecosystems in ecological time. Science 171:385-387

Rosenzweig ML, MacArthur RH (1963) Graphical representation and stability conditions of predator-prey interactions. Am Nat 97(895):209-223

Shulenburger L, Lai Y, Yalinkaya T, Holt RD (1999) Controlling transient chaos to prevent species extinction. Phys Lett A 260:156-161

Steiner CF, Klausmeier CA, Litchman E (2012) Transient dynamics and the destabilizing effects of prey heterogeneity. Ecology 93(3):632-644

Tilman D (1990) Mechanisms of plant competition for nutrients: the elements of a predictive theory of competition. In: Grace J, Tilman D (eds) Perspectives on plant competition. Academic Press, San Diego, pp 117-141

van Rijn PCJ, van Houten YM, Sabelis MW (2002) How plants benefit from providing food to predators even when it is also edible to herbivores. Ecology 83(10):2664-2679 